\title{
The Luminosity Function of Galaxies as Modeled by a Left Truncated Beta Distribution
}

\author{
L. Zaninetti \\ Dipartimento di Fisica, via P.Giuria 1, Turin, Italy \\ Email: zaninetti@ph.unito.it
}

Received 6 December 2013; revised 5 January 2014; accepted 13 January 2014

Copyright (C) 2014 by author and Scientific Research Publishing Inc.

This work is licensed under the Creative Commons Attribution International License (CC BY).

http://creativecommons.org/licenses/by/4.0/

(c) (i) Open Access

\begin{abstract}
A first new luminosity function of galaxies can be built starting from a left truncated beta probability density function, which is characterized by four parameters. In the astrophysical conversion, the number of parameters increases by one, due to the addition of the overall density of galaxies. A second new galaxy luminosity function is built starting from a left truncated beta probability for the mass of galaxies once a simple nonlinear relationship between mass and luminosity is assumed; in this case the number of parameters is six because the overall density of galaxies and a parameter that regulates mass and luminosity are added. The two new galaxy luminosity functions with finite boundaries were tested on the Sloan Digital Sky Survey (SDSS) in five different bands; the results produce a "better fit" than the Schechter luminosity function in two of the five bands considered. A modified Schechter luminosity function with four parameters has been also analyzed.
\end{abstract}

\section{Keywords}

Galaxies: Fundamental Parameters; Galaxies: Statistics; Galaxies: Luminosity Function

\section{Introduction}

The standard luminosity function for galaxies (LF) in the last forty years has been represented by the Schechter LF

$$
\Phi(L) \mathrm{d} L=\left(\frac{\Phi^{*}}{L^{*}}\right)\left(\frac{L}{L^{*}}\right)^{\alpha} \exp \left(-\frac{L}{L^{*}}\right) \mathrm{d} L
$$


where $\alpha$ sets the slope for low values of $L, L^{*}$ is the characteristic luminosity and $\Phi^{*}$ is the normalization, see [1]. This LF is defined in the in the interval $[0, \infty]$ and has replaced other LFs presented by [2]-[5].

The goodness of the fit can be evaluated by the merit function $\chi^{2}$

$$
\chi^{2}=\sum_{j=1}^{n}\left(\frac{L F_{\text {theo }}-L F_{\text {astr }}}{\sigma_{L F_{\text {astr }}}}\right)^{2},
$$

where $n$ is number of data and the two indexes theo and astr stand for theoretical and astronomical, LF respectively. The value of $\chi_{s}^{2}$ which characterizes the Schechter LF for a given astronomical catalog, i.e. the Sloan Digital Sky Survey (SDSS) in five different bands, represents a standard value to improve. Many new LFs has been derived in the last years and we report some examples. A two-component Schechter-like function is, see [6]

$$
\begin{aligned}
& L_{\max }>L>L_{D \text { warf }}: \quad \Phi(L) \mathrm{d} L=\left(\frac{\Phi^{*}}{L^{*}}\right)\left(\frac{L}{L^{*}}\right)^{\alpha} \exp \left(-\frac{L}{L^{*}}\right) \mathrm{d} L, \\
& L_{\text {Dwarf }}>L>L_{\min }: \quad \Phi(L) \mathrm{d} L=\left(\frac{\Phi_{\text {Dwarf }}}{L^{*}}\right)\left(\frac{L}{L_{\text {Dwarf }}}\right)^{\alpha_{\text {Dwarf }}} \mathrm{d} L,
\end{aligned}
$$

where

$$
\Phi_{\text {Dwarf }}=\Phi^{*}\left(\frac{L_{D \text { warf }}}{L^{*}}\right)^{\alpha} \exp \left(-\frac{L_{D \text { warf }}}{L^{*}}\right) .
$$

This two-component LF defined between the maximum luminosity, $L_{\max }$, and the minimum luminosity, $L_{\min }$, has five parameters because two additional parameters have been added: $L_{D \text { warf }}$ which represents the magnitude where dwarfs first dominate over giants and $\alpha_{\text {Dwarf }}$ which regulates the faint slope parameter for the dwarf population.

In order to fit the case of extremely low luminosity galaxies a double Schechter LF with five parameters, see [7], was introduced:

$$
\Phi(L) \mathrm{d} L=\frac{\mathrm{d} L}{L_{*}} \exp \left(-L / L_{*}\right)\left[\phi_{*, 1}\left(\frac{L}{L_{*}}\right)^{\alpha_{1}}+\phi_{*, 2}\left(\frac{L}{L_{*}}\right)^{\alpha_{2}}\right],
$$

where the parameters $\Phi^{*}$ and $\alpha$ which characterize the Schechter LF have been doubled in $\phi_{*, 1}$ and $\phi_{*, 2}$. The strong dependence of LF on different environments such as voids, superclusters and supercluster cores was analyzed by [8] with

$$
F(L) \mathrm{d} L \propto\left(L / L^{*}\right)^{\alpha}\left(1+\left(L / L^{*}\right)^{\gamma}\right)^{(\delta-\alpha) / \gamma} \mathrm{d}\left(L / L^{*}\right),
$$

where $\alpha$ is the exponent at low luminosities $\left(L / L^{*}\right) \ll 1, \delta$ is the exponent at high luminosities $\left(L / L^{*}\right) \gg 1$, $\gamma$ is a parameter of transition between the two power laws, and $L^{*}$ is the characteristic luminosity. Another LF starts from the probability density function (PDF) that models area and volumes of the Voronoi Diagrams and introduces the mass-luminosity relationship, see [9]; in the SDSS case $\chi^{2}>\chi_{S}^{2}$. A last example is represented by three new LFs deduced in the framework of generalized gamma PDF, see [10]; in the SDSS case $\chi^{2}<\chi_{s}^{2}$. All the previous LFs cover the range $[0, \infty]$ and therefore the analysis of finite upper and lower boundaries can be a subject of investigation. Another interesting observational fact is that at low values of luminosity (high absolute magnitude) the observed LF has an approximate constant value. The left truncated beta with scale PDF recently derived, see Equation (34) in [11], satisfies the two issues previously raised. In order to explore the connection between LF and evolution of galaxies see [12]-[18].

Here we analyze in Section 2 a left truncated beta LF for galaxies and in Section 3 a left truncated beta for mass which transforms itself in a LF for galaxies through a nonlinear mass-luminosity relationship. Section 4 reports a recent LF for galaxies which has a finite boundary at the bright end. 


\section{A Linear Mass-Luminosity Relationship}

A new PDF, the left truncated beta with scale has been recently derived, see formula (34) in [11]. Once the random variable $X$ is substituted with the luminosity $L$ we obtain a new $L F$ for galaxies, $\Psi$,

$$
\Psi(L) \mathrm{d} L=K_{L} \Psi^{*} L^{\alpha-1}\left(L_{b}-L\right)^{\beta-1} \mathrm{~d} L,
$$

where $\Psi^{*}$ is a normalization factor which defines the overall density of galaxies, a number per cubic Mpc. The constant $K_{L}$ is

$$
\begin{aligned}
& K_{L}=\frac{A}{B} \\
& A=-\alpha \Gamma(\alpha+\beta) \\
& B=L_{b}^{\beta-1}{ }_{2} F_{1}\left(\alpha,-\beta+1 ; 1+\alpha ; \frac{L_{a}}{L_{b}}\right) L_{a}^{\alpha} \Gamma(\alpha+\beta)-L_{b}^{\beta+\alpha-1} \Gamma(1+\alpha) \Gamma(\beta),
\end{aligned}
$$

and $L_{a}, L_{b}$ are the lower, upper values in luminosity and ${ }_{2} F_{1}(a, b ; c ; z)$ is the regularized hypergeometric function [19]-[23]. The hypergeometric function with real arguments is implemented in the FORTRAN subroutine MHYGFX in [24]. The averaged luminosity, $\langle L\rangle$, is:

$$
\langle L\rangle=K_{L} \Psi^{*} \frac{A_{N}}{B_{D}},
$$

where

$$
\begin{aligned}
& A_{N}=-L_{b}^{\beta-1} L_{a}^{1+\alpha}{ }_{2} F_{1}\left(-\beta+1,1+\alpha ; 2+\alpha ; \frac{L_{a}}{L_{b}}\right) \Gamma(1+\alpha+\beta)+L_{b}^{\alpha+\beta} \Gamma(2+\alpha) \Gamma(\beta) \\
& B_{D}=(1+\alpha) \Gamma(1+\alpha+\beta) .
\end{aligned}
$$

The relationships connecting the absolute magnitude $M, M_{a}$ and $M_{b}$ of a galaxy to respective luminosities are:

$$
\frac{L}{L_{\odot}}=10^{0.4\left(M_{\odot}-M\right)}, \frac{L_{a}}{L_{\odot}}=10^{0.4\left(M_{\odot}-M_{a}\right)}, \frac{L_{b}}{L_{\odot}}=10^{0.4\left(M_{\odot}-M_{b}\right)},
$$

where $M_{\odot}$ is the absolute magnitude of the sun in the considered band. The LF in magnitude is

$$
\begin{aligned}
\Psi(M) \mathrm{d} M= & -K_{M} \Psi^{*} 0.4\left(10^{-0.4 a m+0.4 M_{\odot}}\right)^{\alpha-1} \times\left(10^{-0.4 M_{b}+0.4 M_{\odot}}-10^{-0.4 a m+0.4 M_{\odot}}\right)^{\beta-1} \\
& \times 10^{-0.4 a m+0.4 M_{\odot}} \ln (10) \mathrm{d} M,
\end{aligned}
$$

where

$$
\begin{aligned}
K_{M}= & \frac{A_{M}}{B_{M}} \\
A_{M}= & \alpha \Gamma(\alpha+\beta) \\
B_{M}= & -\left(10^{-0.4 M_{b}+0.4 M_{\odot}}\right)^{\beta-1} \times{ }_{2} F_{1}\left(\alpha,-\beta+1 ; 1+\alpha ; \frac{10^{-0.4 M_{a}+0.4 M_{\odot}}}{10^{-0.4 M_{b}+0.4 M_{\odot}}}\right) \\
& \times\left(10^{-0.4 M_{a}+0.4 M_{\odot}}\right)^{\alpha} \Gamma(\alpha+\beta)+\left(10^{-0.4 M_{b}+0.4 M_{\odot}}\right)^{\beta+\alpha-1} \Gamma(1+\alpha) \Gamma(\beta) .
\end{aligned}
$$

This data-oriented LF contains the five parameters $\alpha, \beta, M_{a}, M_{b}, \Psi^{*}$ which can be derived from the operation of fitting the observational data and $M_{\odot}$ which characterize the considered band. The number of variables can be reduced to three once $M_{a}$ and $M_{b}$ are identified with the maximum and the minimum absolute magnitude of the considered sample. The numerical analysis points toward value of $\alpha \approx 0$. Once as an example we fix $\alpha=0.01$ we have only to find the two parameters $\beta$ and $\Psi^{*}$. The test on the new LF were performed on the data of the Sloan Digital Sky Survey (SDSS) which has five bands $u^{*}, g^{*}, r^{*}, i^{*}$, and $z^{*}$, see [25]. 
Table 1 reports the bolometric magnitude, the numerical values obtained for the new LF, the number, $N$, of the astronomical sample, the $\chi^{2}$ of the new LF, the reduced $\chi^{2}, \chi_{\text {red }}^{2}$, of the new LF, the $\chi_{s}^{2}$ of the Schechter LF, the reduced $\chi^{2}, \chi_{s, \text { red }}^{2}$, of Schechter LF and the $\chi_{s}^{2}$ computed as in [25] for the five bands here considered.

\section{A Non Linear Mass-Luminosity Relationship}

We assume that the mass of the galaxies, $\mathcal{M}$, is distributed with a left truncated PDF as represented by formula (34) in [11]. The distribution for the mass is $\Psi\left(\mathcal{M} ; \mathcal{M}_{a}, \mathcal{M}_{b}, \alpha, \beta\right)$ where $\mathcal{M}_{a}$ is the lower mass and $\mathcal{M}_{b}$ is the upper mass. The mass is supposed to scale as

$$
\mathcal{M}=L^{\frac{1}{c}},
$$

where $c$ is the parameter that regulates the non linear mass-luminosity relationship. The differential of the mass is

$$
\mathrm{d} \mathcal{M}=\frac{L^{\frac{1}{c}-1}}{C} \mathrm{~d} L .
$$

As a consequence the LF representing the non linear mass-luminosity relationship is

$$
\Psi_{M L}(L) \mathrm{d} L=K_{M L} \Psi_{M L}^{*} \frac{\left(L^{c^{-1}}\right)^{\alpha-1}\left(L_{b}^{c^{-1}}-L^{c^{-1}}\right)^{\beta-1} L^{c^{-1}}}{c L} \mathrm{~d} L,
$$

where

$$
\begin{aligned}
& K_{M L}=\frac{A_{M L}}{B_{M L}} \\
& A_{M L}=-\alpha \Gamma(\alpha+\beta) \\
& B_{M L}=\left(L_{b}^{c^{-1}}\right)^{\beta-1}{ }_{2} F_{1}\left(\alpha,-\beta+1 ; 1+\alpha ; \frac{L_{a}^{c^{-1}}}{L_{b}^{c^{-1}}}\right)\left(L_{a}^{c^{-1}}\right)^{\alpha} \Gamma(\alpha+\beta)-\left(L_{b}^{c^{-1}}\right)^{\beta-1+\alpha} \Gamma(1+\alpha) \Gamma(\beta) .
\end{aligned}
$$

The averaged luminosity, $\langle L\rangle$, for the non linear mass-luminosity relationship is:

Table 1. Parameters of fits in SDSS Galaxies of LF as represented by formula (10) in which the number of free parameters is 5.

\begin{tabular}{cccccc}
\hline parameter & $u^{*}$ & $g^{*}$ & $r^{*}$ & $i^{*}$ & $z^{*}$ \\
\hline$M_{\odot}$ & 6.38 & 5.07 & 4.62 & 4.52 & 4.48 \\
$M_{a}$ & -15.90 & -16.56 & -16.81 & -17.27 & -17.56 \\
$M_{b}$ & -21.48 & -22.95 & -24.03 & -24.45 & -24.75 \\
$\Psi^{*}\left[\mathrm{~h}^{3} \cdot \mathrm{Mpc}^{-3}\right]$ & 0.040 & 0.044 & 0.042 & 0.038 & 0.038 \\
$\alpha$ & 0.01 & 0.01 & 0.01 & 0.01 & 0.01 \\
$\beta$ & 23.44 & 23.83 & 27.88 & 27.56 & 27.36 \\
$N$ & 483 & 599 & 674 & 709 & 740 \\
$\chi^{2}$ & 570 & 1032 & 4342 & 3613 & 6252 \\
$\chi_{\text {red }}^{2}$ & 1.19 & 1.73 & 6.49 & 5.13 & 8.5 \\
$\chi_{s}^{2}$ & 330 & 753 & 2260 & 2282 & 3245 \\
$\chi_{S, \text { red }}^{2}$ & 0.689 & 1.263 & 3.368 & 3.232 & 4.403 \\
$\chi^{2}-$ Blanton 2003 & 341 & 756 & 2276 & 2283 & 3262 \\
\hline
\end{tabular}




$$
\langle L\rangle=K_{M L} \Psi^{*} \frac{A_{N M}}{B_{D M}}
$$

where

$$
\begin{aligned}
A_{\mathrm{NM}}= & L_{b}^{\frac{c+\alpha+\beta-1}{c}} \Gamma(c+\alpha) \Gamma(\beta) c+L_{b}{ }^{\frac{c+\alpha+\beta-1}{c}} \Gamma(c+\alpha) \Gamma(\beta) \alpha \\
& -L_{b}^{\frac{\beta-1}{c}}{ }_{2} F_{1}\left(-\beta+1, c+\alpha ; 1+\alpha+c ; L_{a}^{c^{-1}} L_{b}^{c^{-1}}\right) \times L_{a}^{\frac{c+\alpha}{c}} \Gamma(c+\alpha+\beta) \\
B_{\mathrm{DM}}= & \Gamma(c+\alpha+\beta)(c+\alpha) .
\end{aligned}
$$

The LF in magnitude for the non linear mass-luminosity relationship is

$$
\begin{aligned}
& \Psi_{M L}(M) \mathrm{d} M=-K_{M M L} \Psi^{*} \\
& {\left[0.4\left(\left(10^{-0.4 a m+0.4 M_{\odot}}\right)^{c^{-1}}\right)^{\alpha-1}\left(\left(10^{-0.4 M_{b}+0.4 M_{\odot}}\right)^{c^{-1}}-\left(10^{-0.4 a m+0.4 M_{\odot}}\right)^{c^{-1}}\right)^{\beta-1}\left(10^{-0.4 a m+0.4 M_{\odot}}\right)^{c^{-1}} \ln (10)\right] / c,}
\end{aligned}
$$

where

$$
\begin{aligned}
K_{M M L}= & \frac{A_{M M L}}{B_{M M L}} \\
A_{M M L}= & \alpha \Gamma(\alpha+\beta) \\
B_{M M L}= & -\left(\left(10^{-0.4 M_{b}+0.4 M_{\odot}}\right)^{c^{-1}}\right)^{\beta-1} \times{ }_{2} F_{1}\left(\alpha,-\beta+1 ; 1+\alpha ; \frac{\left(10^{-0.4 M_{a}+0.4 M_{\odot}}\right)^{c^{-1}}}{\left(10^{-0.4 M_{b}+0.4 M_{\odot}}\right)^{c^{-1}}}\right) \\
& \times\left(\left(10^{-0.4 M_{b}+0.4 M_{\odot}}\right)^{c^{-1}}\right)^{\alpha} \Gamma(\alpha+\beta)+\left(\left(10^{-0.4 M_{b}+0.4 M_{\odot}}\right)^{c^{-1}}\right)^{\beta-1+\alpha} \Gamma(1+\alpha) \Gamma(\beta) .
\end{aligned}
$$

\begin{tabular}{|c|c|c|c|c|c|}
\hline parameter & $u^{*}$ & $g^{*}$ & $r^{*}$ & $i^{*}$ & $Z^{*}$ \\
\hline$M_{a}$ & -15.38 & -16.2 & -16.59 & -16.99 & -17.41 \\
\hline$M_{b}$ & -22.3 & -23.31 & -24.68 & -25.02 & -25.34 \\
\hline$\Psi^{*}\left[\mathrm{~h}^{3} \cdot \mathrm{Mpc}^{-3}\right]$ & 0.048 & 0.045 & 0.046 & 0.041 & 0.043 \\
\hline$\alpha$ & 1.0 & 0.16 & 0.16 & 0.3 & 0.09 \\
\hline$\beta$ & 33.05 & 30.16 & 33.92 & 31.02 & 32.41 \\
\hline$C$ & 1.52 & 1.1 & 1.25 & 1.31 & 1.23 \\
\hline$\chi^{2}$ & 314 & 866 & 2568 & 2174 & 3325 \\
\hline$\chi_{\text {red }}^{2}$ & 0.66 & 1.46 & 3.84 & 3.09 & 4.53 \\
\hline
\end{tabular}

Table 2 reports the numerical values obtained for the new nonlinear LF as represented by Equation (17), the $\chi^{2}$ of the new LF and the $\chi_{\text {red }}^{2}$ of the new LF in the five bands here considered.

The Schechter LF, the new LF represented by formula (17) and the data of SDSS are reported in Figure 1-5, where bands $u^{*}, g^{*}, r^{*}, i^{*}$, and $z^{*}$ are considered.

A discussion on the distribution of $M / L$ in all photometric bands can be found in [26].

\section{A Modified Schechter LF}

An example of modified Schechter LF is represented by the following function

Table 2. Parameters of fits in SDSS Galaxies of LF as represented by our non linear LF (17) formula (10) in which the number of free parameters is 6. 


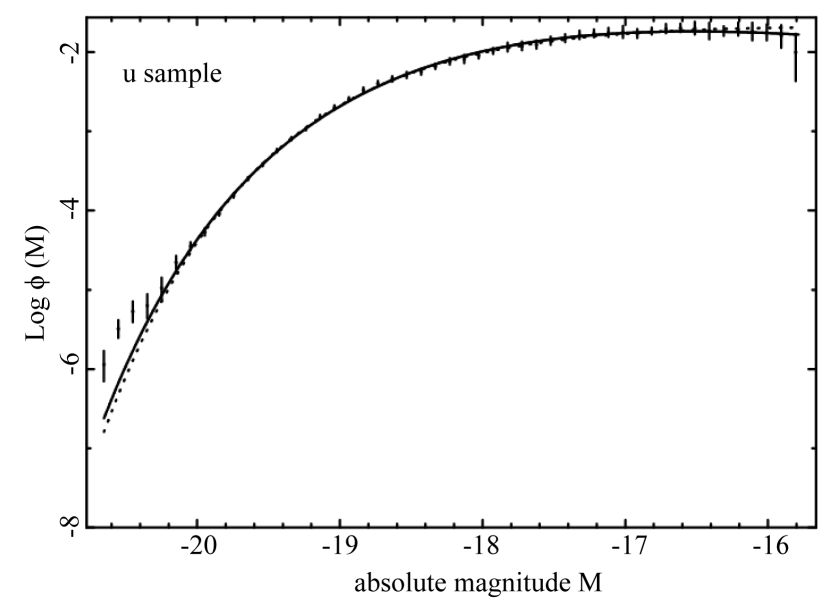

Figure 1. The LF data of SDSS $\left(u^{*}\right)$ are represented with error bars. The continuous line fit represents our non linear LF (17) and the dotted line represents the Schechter LF.

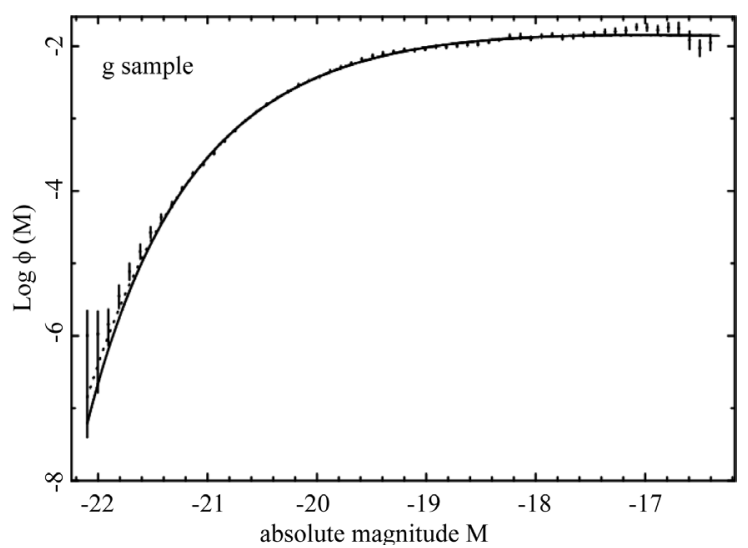

Figure 2. The luminosity function data of SDSS $\left(g^{*}\right)$ are represented with error bars. The continuous line fit represents our non linear LF (10) and the dotted line represents the Schechter LF.

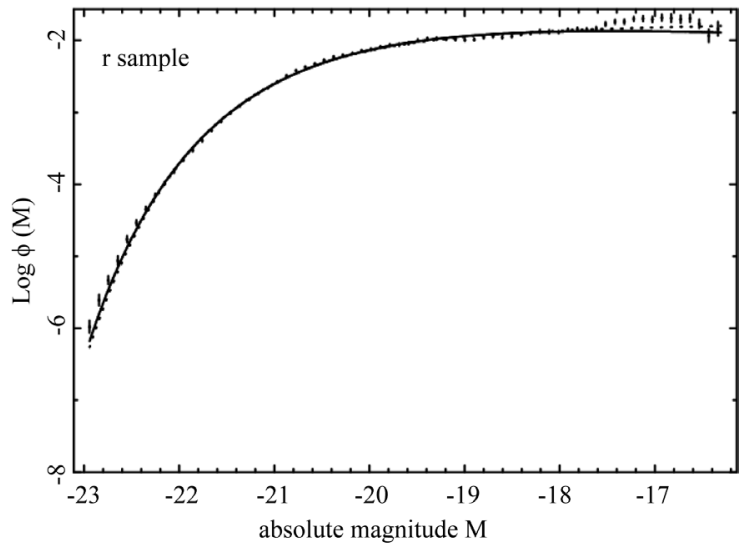

Figure 3. The luminosity function data of SDSS $\left(r^{*}\right)$ are represented with error bars. The continuous line fit represents our non linear LF (10) and the dotted line represents the Schechter LF. 


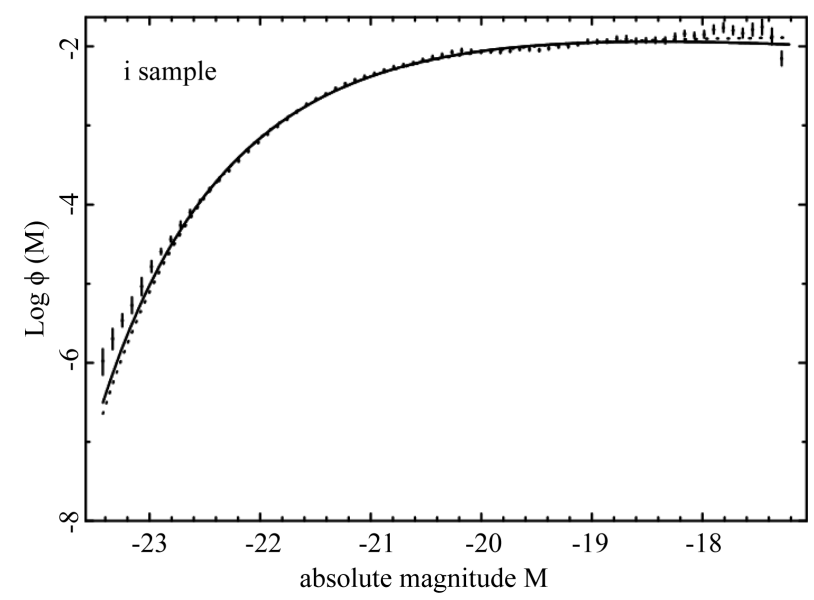

Figure 4. The luminosity function data of $\operatorname{SDSS}\left(i^{*}\right)$ are represented with error bars. The continuous line fit represents our non linear LF (10) and the dotted line represents the Schechter LF.

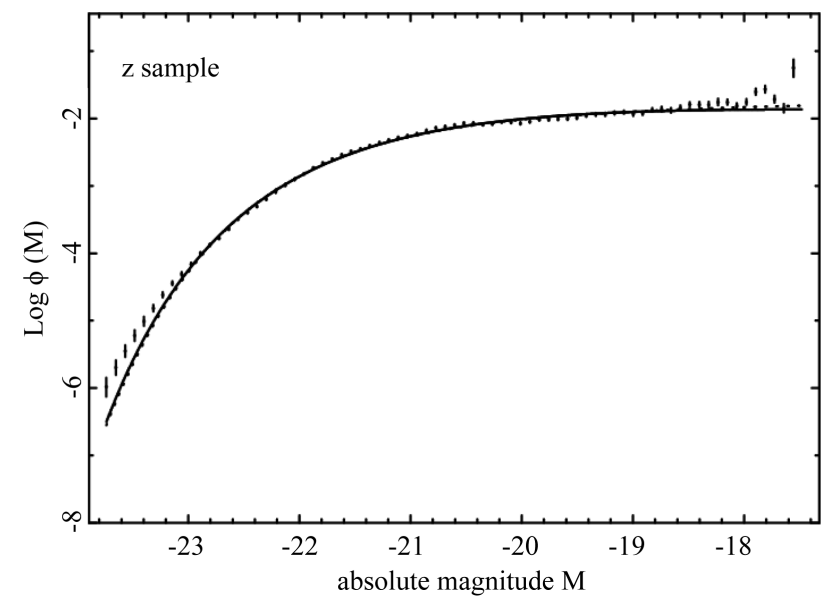

Figure 5. The luminosity function data of $\operatorname{SDSS}\left(z^{*}\right)$ are represented with error bars. The continuous line fit represents our non linear LF (10) and the dotted line represents the Schechter LF.

$$
\Phi_{B}(L) \mathrm{d} L=\frac{\Phi^{*}\left(\frac{L}{L^{*}}\right)^{\alpha}\left(1-\frac{(\eta-1) L}{L^{*}}\right)^{\frac{1}{\eta-1}}}{L^{*}} \mathrm{~d} L
$$

where $\eta$ is a new parameter, see [27]. This new LF in the case of $\eta<1$ is defined in the range $0<L<L_{\max }$ where $L_{\max }=\frac{L^{*}}{\eta-1}$ and therefore has a natural upper boundary which is not infinity. In the limit $\lim _{\eta \rightarrow 1} \Phi_{B}(L)$ the Schechter LF is obtained. In the case of $\eta>1$ the average value is

$$
\langle L\rangle=\frac{\Phi^{*} L^{*} \Gamma(3+\alpha) \Gamma\left(1+(\eta-1)^{-1}\right)(\eta-1)^{-\alpha-2}}{\Gamma\left(3+\alpha+(\eta-1)^{-1}\right)(\alpha+2)},
$$

and in the case of $\eta<1$ the average value is 


$$
\langle L\rangle=\frac{\Phi^{*} L^{*}(-\eta+1)^{-2-\alpha} \Gamma\left(-\frac{-1+2 \eta+\alpha \eta-\alpha}{\eta-1}\right) \Gamma(2+\alpha)}{\Gamma\left(-(\eta-1)^{-1}\right)} .
$$

The distribution in magnitude is:

$$
\Phi(M) \mathrm{d} M=0.921 \Phi^{*} 10^{0.4\left(M^{*}-M\right)(\alpha+1)}\left(1-(\eta-1) 10^{0.4\left(M^{*}-M\right)}\right)^{\frac{1}{\eta-1}} \mathrm{~d} M .
$$

As an example the Schechter LF, the modified Schechter LF represented by formula (22) and the data of SDSS are reported in Figure 6 for the $u^{*}$ band and Table 3 reports the results of the fits in all the five bands of SDSS.

\section{Conclusions}

Motivations The observational fact that the LF for galaxies spans from a minimum to a maximum value in

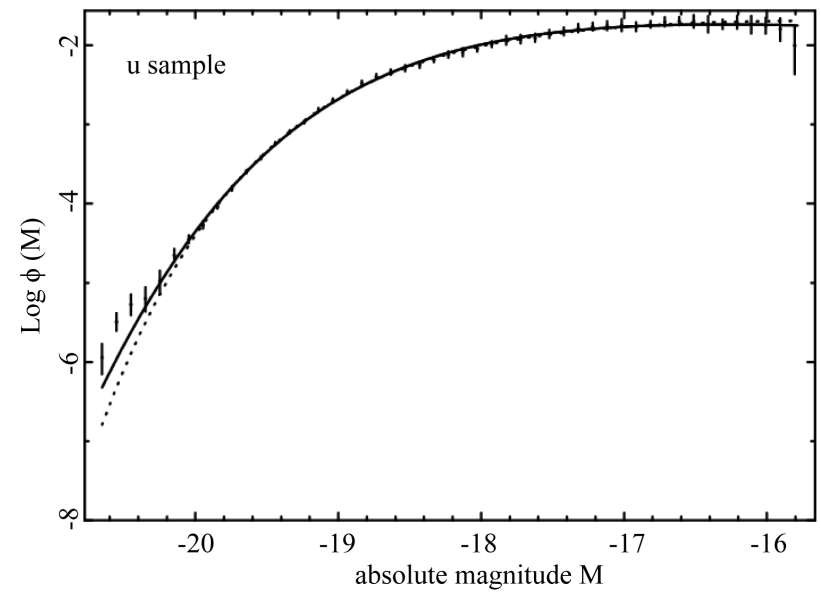

Figure 6. The luminosity function data of SDSS $\left(u^{*}\right)$ are represented with error bars. The continuous line fit represents the modified Schechter LF (22) and the dotted line represents the Schechter LF.

Table 3. Parameters of fits in SDSS Galaxies of the modified Schechter LF as represented by formula (22) in which the

\begin{tabular}{|c|c|c|c|c|c|}
\hline parameter & $u^{*}$ & $g^{*}$ & $r^{*}$ & $i^{*}$ & $z^{*}$ \\
\hline$M^{*}$ & -17.68 & -19.36 & -20.30 & -20.54 & -20.92 \\
\hline$\Phi^{*}\left[\mathrm{~h}^{3} \cdot \mathrm{Mpc}^{-3}\right]$ & 0.037 & 0.022 & 0.016 & 0.018 & 0.017 \\
\hline$\alpha$ & -0.721 & -0.874 & -0.983 & -0.856 & -0.942 \\
\hline$\eta$ & 0.959 & 0.994 & 0.966 & 0.938 & 0.94 \\
\hline$N$ & 483 & 599 & 674 & 709 & 740 \\
\hline$\chi^{2}$ & 252 & 749 & 1944 & 1498 & 2409 \\
\hline$\chi_{\text {red }}^{2}$ & 0.526 & 1.25 & 2.9 & 2.12 & 3.27 \\
\hline$\chi_{s}^{2}$ & 330 & 753 & 2260 & 2282 & 3245 \\
\hline$\chi_{S, \text { red }}^{2}$ & 0.689 & 1.263 & 3.368 & 3.232 & 4.403 \\
\hline$\chi^{2}-$ Blanton 2003 & 341 & 756 & 2276 & 2283 & 3262 \\
\hline
\end{tabular}
number of free parameters is 4 . 
absolute magnitude makes attractive the exploration of the left truncated beta LF. We derived two LFs adopting the framework of the linear M-L relationship, see Equation (10), and the framework of the non linear M-L relationship , see Equation (17).

\subsection{Goodness of Fit Tests}

We have computed $\chi^{2}$ and $\chi_{\text {red }}^{2}$ for two LFs here derived and compared the results with the Schechter LF, see Tables 1 and 2. In the linear M-L case $\chi^{2}$ and $\chi_{\text {red }}^{2}$ are greater of that of the Schechter LF. In the non linear M-L case $\chi^{2}$ and $\chi_{\text {red }}^{2}, u^{*}$ and $i^{*}$, are smaller in two bands over five in respect to those of the Schechter LF. The non linear case suggests a power law behavior with an exponent $1.1<c<1.52$ and therefore M-L relationship has an exponent greater than 1 but smaller than 4 , the theoretical value of the stars.

\subsection{Modified Schechter LF}

The modified Schechter LF when $\eta<1$, which is the case of SDSS, has by definition an upper boundary, see 4. The $\chi^{2}$ and $\chi_{\text {red }}^{2}$ are smaller in five bands over five in respect to those of the Schechter LF. This goodness of fit is due to the flexibility introduced by the fourth parameter $\eta$ as outlined in [27].

\subsection{Physical Motivations}

The Schechter LF is motivated by the Press-Schechter formalism on the self-similar gravitational condensation. Here conversely we limited ourself to fix a lower and an upper bound on the mass of a galaxy and we translated this requirement in a PDF for mass and then in LF. Finite-size effects on the luminosity of SDSS galaxies has also been explored in [28].

\section{References}

[1] Schechter, P. (1976) An Analytic Expression for the Luminosity Function for Galaxies. Astrophysical Journal, 203, 297-306. http://dx.doi.org/10.1086/154079

[2] Zwicky, F. (1957) Morphological Astronomy. Springer, Berlin. http://dx.doi.org/10.1007/978-3-642-87544-1

[3] Kiang, T. (1961) The Galaxian Luminosity Function. Monthly Notices of the Royal Astronomical Society, $122,263$.

[4] Abell, G.O. (1965) Clustering of Galaxies. Annual Review of Astronomy and Astrophysics, 3, 1-22.

[5] Arakelyan, M.A. and Kalloglyan, A.T. (1970) The Luminosity Function of Field Galaxies. Soviet Astronomy, 13, 953.

[6] Driver, S.P. and Phillipps, S (1996) Is the Luminosity Distribution of Field Galaxies Really Flat? Astrophysical Journal, 469, 529. http://dx.doi.org/10.1086/177801

[7] Blanton, M.R., Lupton, R.H., Schlegel, D.J., Strauss, M.A., Brinkmann, J., Fukugita, M. and Loveday, J. (2005)The Properties and Luminosity Function of Extremely Low Luminosity Galaxies. Astrophysical Journal, 631, 208.

[8] Tempel, E., Einasto, J., Einasto, M., Saar, E. and Tago, E. (2009) Anatomy of Luminosity Functions: The 2dFGRS Example. Astronomy \& Astrophysics, 495, 37.

[9] Zaninetti, L. (2008) A New Luminosity Function for Galaxies as Given by the Mass-Luminosity Relationship. The Astronomical Journal, 135, 1264-1275.

[10] Zaninetti, L. (2010) The Luminosity Function of Galaxies as Modelled by the Generalized Gamma Distribution. Acta Physica Polonica B, 41, 729.

[11] Zaninetti, L. (2013) The Initial Mass Function Modeled by a Left Truncated Beta Distribution. Astrophysical Journal, 765, 128.

[12] Van den Bosch, F.C., Yang, X. and Mo, H.J. (2003) Linking Early- and Late-Type Galaxies to Their Dark Matter Haloes. Monthly Notices of the Royal Astronomical Society, 340, 771. http://dx.doi.org/10.1046/j.1365-8711.2003.06335.x

[13] Yang, X., Mo, H.J. and van den Bosch, F.C. (2003) Constraining galaxy formation and cosmology with the conditional luminosity function of galaxies. Monthly Notices of the Royal Astronomical Society, 339, 1057. http://dx.doi.org/10.1046/j.1365-8711.2003.06254.x

[14] Cooray, A. and Cen, R. (2005) The Rise of Dwarfs and the Fall of Giants: Galaxy Formation Feedback Signatures in the Halo Satellite Luminosity Function. Astrophysical Journal, 633, L69.

[15] Cooray, A. and Milosavljevic, M. (2005) What is L*? Anatomy of the Galaxy Luminosity Function. Astrophysical Journal, 627, L89. 
[16] Cooray, A. and Milosavljevic, M. (2005) Dissipationless Merging and the Assembly of Central Galaxies. Astrophysical Journal, 627, L85.

[17] Tinker, J.L., Weinberg, D.H., Zheng, Z. and Zehavi, I. (2005) On the Mass-to-Light Ratio of Large-Scale Structure. Astrophysical Journal, 631, 41.

[18] Tinker, J.L., Norberg, P., Weinberg, D.H. and Warren, M.S. (2007) On the Luminosity Dependence of the Galaxy Pairwise Velocity Dispersion. Astrophysical Journal, 659, 877.

[19] Abramowitz, M. and Stegun, I.A. (1965) Handbook of Mathematical Functions with Formulas, Graphs, and Mathematical Tables. Dover, New York.

[20] Von Seggern, D. (1992) CRC Standard Curves and Surfaces. CRC, New York.

[21] Thompson, W.J. (1997) Atlas for computing mathematical functions. Wiley-Interscience, New York.

[22] Gradshteyn, I.S. and Ryzhik, I.M. and Jeffrey, A. and Zwillinger, D. (2007) Table of Integrals, Series, and Products. Academic Press, New York.

[23] Olver, F.W.J., Lozier, D. W., Boisvert R.F. and Clark C.W. (2010) NIST Handbook of Mathematical Functions. Cambridge University Press, Cambridge.

[24] Zhang, S. and Jin, J. (1996) Computation of Special Functions. Wiley-Interscience, New York.

[25] Blanton, M.R., Hogg, D.W., Bahcall, N.A., Brinkmann, J. and Britton, M. (2003) The Galaxy Luminosity Function and Luminosity Density at Redshift z = 0.1. Astrophysical Journal, 592, 819.

[26] Kauffmann, G., Heckman, T.M., White, S.D.M., Charlot, S. and Tremonti, C. (2003) Stellar masses and star formation histories for $10^{5}$ galaxies from the Sloan Digital Sky Survey. Monthly Notices of the Royal Astronomical Society, 341, 33-53.

[27] Alcaniz, J.S. and Lima, J.A.S. (2004) Galaxy Luminosity Function: A New Analytic Expression. Brazilian Journal of Physics, 34, 455. http://dx.doi.org/10.1590/S0103-97332004000300026

[28] Taghizadeh-Popp, M., Ozogány, K., Rácz, Z., Regoes, E. and Szalay, A.S. (2012) Distribution of Maximal Luminosity of Galaxies in the Sloan Digital Sky Survey. Astrophysical Journal, 759, 100. 